\title{
Biofilm formation and migration on ventricular assist device drivelines
}

\author{
Yue Qu, PhD, ${ }^{\text {a,b }}$ David McGiffin, $\mathrm{MD},{ }^{\mathrm{c}}$ Christina Kure, $\mathrm{PhD},{ }^{\mathrm{c}}$ Berkay Ozcelik, $\mathrm{PhD},{ }^{\mathrm{d}}$ \\ John Fraser, MD, PhD, ${ }^{\mathrm{e}}$ Helmut Thissen, $\mathrm{PhD},{ }^{\mathrm{d}}$ and Anton Y. Peleg, $\mathrm{MD}, \mathrm{PhD}^{\mathrm{a}, \mathrm{b}}$
}

\section{ABSTRACT}

Objectives: Driveline infections remain an important complication of ventricular assist device therapy, with biofilm formation being a major contributor. This study aimed to elucidate factors that govern biofilm formation and migration on clinically relevant ventricular assist device drivelines.

Methods: Experimental analyses were performed on HeartWare HVAD (HeartWare International Inc, Framingham, Mass) drivelines to assess surface chemistry and biofilm formation. To mimic the driveline exit site, a drip-flow biofilm reactor assay was used. To mimic a subcutaneous tissue environment, a tunnel-based interstitial biofilm assay was developed. Clinical HVAD drivelines explanted at the time of cardiac transplantation were also examined by scanning electron microscopy.

Results: Common causative pathogens of driveline infections were able to adhere to the smooth and velour sections of the HVAD driveline and formed robust biofilms in the drip-flow biofilm reactor; however, Pseudomonas aeruginosa and Candida albicans had greater biomass. Biofilm migration within the interstitial driveline tunnel was evident for Staphylococcus epidermidis, Staphylococcus aureus, and $C$ albicans, but not $P$ aeruginosa. Biofilm formation by staphylococci was 500 to 10,000 times higher in the tunnel-based model compared with our exit site model. The 3-dimensional structure of the driveline velour and the use of silicone adhesive in driveline manufacturing were found to promote biofilm growth, and explanted patient drivelines demonstrated inadequate tissue in-growth along the entire velour with micro-gaps between velour fibers.

Conclusions: This work highlights the predilection of pathogens to different parts of the driveline, the importance of the subcutaneous tunnel to biofilm formation and migration, and the presence of micro-gaps in clinical drivelines that could facilitate invasive driveline infections. ( $\mathrm{J}$ Thorac Cardiovasc Surg 2020;159:491-502)

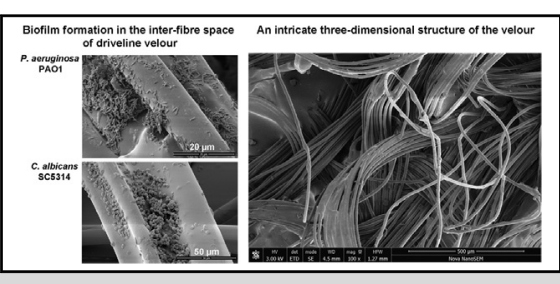

The 3-dimensional structure of velour provides a scaffold for biofilm growth.

\section{Central Message}

Pathogens variably adhere and form biofilms on the smooth and velour sections of drivelines, with silicone adhesive glue and the subcutaneous tunnel augmenting the formation and migration of biofilms.

\section{Perspective}

This study identified key factors involved in biofilm formation and migration on VAD drivelines, including surface chemistry and design of the driveline, and the presence of a nutrient-rich subcutaneous tunnel. These findings lay the foundation for preventative strategies, including improved tissue integration into the velour, altered driveline manufacturing, and surface-attached antimicrobials.

See Commentary page 503.

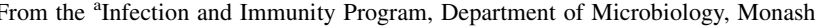
Biomedicine Discovery Institute, Monash University, Clayton, Australia; ${ }^{\mathrm{b}}$ Department of Infectious Diseases, The Alfred Hospital and Central Clinical School, Monash University, Melbourne, Australia; ${ }^{\mathrm{c} D e p a r t m e n t ~ o f ~ C a r d i o t h o r a c i c ~ S u r g e r y, ~}$ The Alfred and Monash University, Melbourne, Australia; ${ }^{\mathrm{d}}$ CSIRO Manufacturing, Clayton, Australia; and ${ }^{\mathrm{e}}$ Adult Intensive Care Service, The Prince Charles Hospital, Brisbane, Australia.

This study was financially supported by The Alfred Hospital Foundation; Monash Institute of Medical Engineering, Monash University; The National Health and Medical Research Council (1079421) CRE ACTIONS; and Australian NHMRC Practitioner Fellowship (APP1117940).

Received for publication Sept 16, 2018; revisions received Feb 21, 2019; accepted for publication Feb 22, 2019; available ahead of print April 5, 2019.

Address for reprints: Anton Y. Peleg, MD, PhD, Department of Infectious Diseases, The Alfred Hospital and Central Clinical School, Monash University, Melbourne, Australia (E-mail: anton.peleg@monash.edu).

$0022-5223 / \$ 36.00$

Copyright (C) 2019 by The American Association for Thoracic Surgery

https://doi.org/10.1016/j.jtcvs.2019.02.088
}

One of the long-term adverse events of ventricular assist devices (VADs) is the development of a driveline infection, contributing substantially to patient morbidity and mortality and imposing a significant healthcare economic burden. The driveline is a percutaneous cable traversing a subcutaneous tunnel and connecting the pump with the external controller unit, conducting power, controller algorithms, and telemetric data. A typical HVAD driveline (HeartWare

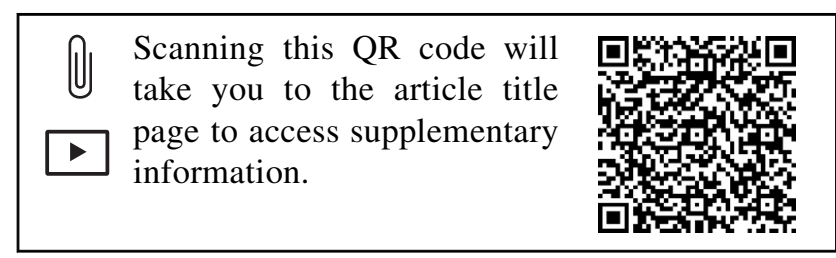



Abbreviations and Acronyms
PBS = phosphate-buffered saline
PDMS $=$ polydimethylsiloxane
SEM = scanning electron microscopy
VAD $=$ ventricular assist device
XPS $=$ X-ray photoelectron spectroscopy

International Inc, Framingham, Mass) consists of a segment of smooth tubing made of polyurethane and another segment of tubing wrapped with polystyrene velour positioned in the subcutaneous tunnel. The velour was designed to stabilize the driveline by anchoring it within the tissue tunnel.

Approximately $10 \%$ to $20 \%$ of drivelines become infected, ${ }^{2-4}$ with microbial biofilm formation being a major causative factor. Biofilms are communities of microorganisms irreversibly attached to a surface that have a protective extracellular matrix and altered metabolic rate, leading to resistance to antimicrobial and host immune attack. ${ }^{5}$ It has been proposed that VAD-associated infections start with microbial adherence and colonization of the external driveline surface, with subsequent biofilm formation leading to the initiation of infection. $^{6,7}$ Migration of microorganisms along the driveline surface or dislodgment of microorganisms from the biofilm can lead to more invasive infections involving the bloodstream or the pump. ${ }^{7,8}$ Despite its crucial role in VAD infections and the high infection rate over the lifetime of these devices, biofilm formation on VAD drivelines has not been studied to a great extent. ${ }^{7}$ There is a lack of knowledge regarding the propensity of microorganisms to attach and form biofilms to the different components of the driveline (smooth and velour sections) and the factors driving biofilm migration along the driveline that may lead to more invasive infections.

Elimination of drivelines with the introduction of transcutaneous energy transfer systems is the ultimate solution to driveline infections, but this technology is still under development. Prevention of driveline infections using antimicrobials has shown low efficacy and is accompanied by the risk of developing antimicrobial resistance. ${ }^{9}$ The objectives of this study were to comprehensively assess biofilm formation on clinically relevant drivelines using models that mimic environmental conditions encountered by an implanted driveline and to quantify biofilm migration. Furthermore, we assessed explanted patient drivelines using scanning electron microscopy (SEM). These data provide crucial insights for the development of novel preventative strategies for one of the most troublesome complications of mechanical circulatory support.

\section{MATERIALS AND METHODS Microorganisms and Driveline Materials for In Vitro Studies}

Four reference clinical strains were selected for this study, including Staphylococcus epidermidis RP62A (ATCC 35984), Staphylococcus aureus ATCC 25923, Pseudomonas aeruginosa PAO1, and Candida albicans SC5314. These microorganisms were selected because they represent the most common microbial species causing VAD-associated infections. HVAD drivelines (US) were donated by HeartWare International Inc (Sydney, Australia). To study their surface chemistry (using X-ray photoelectron spectroscopy [XPS] analysis methods described in the Appendix E1) and interaction with microorganisms, both the smooth tube and the velour of drivelines were cut into sections as described in the Appendix E1.

\section{Early Driveline Microbial Adherence Assay}

An in vitro adherence assay was developed to evaluate early microbial adherence to driveline materials, a critical step of biofilm formation. A sterile blank filter disk ( $6 \mathrm{~mm}$ ) was saturated with $25 \mu \mathrm{L}$ of microbial suspensions prepared in Muller-Hinton broth (for $S$ epidermidis, $S$ aureus, and Pseudomonas aeruginosa, optical density at $600 \mathrm{~nm}=0.1$ ) or Roswell Park Memorial Institute medium (1640, for $C$ albicans, optical density at $600 \mathrm{~nm}=1.0) .{ }^{10}$ Driveline cut-outs were placed on the saturated filter disks, with the convex surface directly contacting the filter disk (Figure 1, A). The set-up was then incubated at $37^{\circ} \mathrm{C}$ in a humidified chamber for 2 hours. The convex surface was carefully washed 3 times using sterile phosphate-buffered saline (PBS). The washed driveline section was then placed in a sterile Eppendorf tube containing $1 \mathrm{~mL}$ of sterile PBS and sonicated for 10 minutes using a sonication bath $(42 \mathrm{KHz}, \mathrm{BRAN}-$ SON 1510), followed by vortexing at the highest speed for 2 minutes (30 seconds $\times 4$ ). The suspensions were serially diluted and plated on nutrient agar plates or yeast-peptone dextrose agar plates for viable counts.

\section{Drip-Flow Biofilm Reactor Assay}

A drip-flow biofilm reactor to assess biofilm formation in chronic wound infections has been developed. ${ }^{11,12}$ Reactor apparatus was assembled as described by Goeres and colleagues ${ }^{13}$ (Figure 1,B). ${ }^{13}$ Driveline cut-outs with attached microorganisms, prepared as per the microbial adherence assay, were placed on a filter membrane in the biofilm incubation chamber (Figure 1, B). Further methods are described in the Appendix E1.

\section{Tunnel-Based Interstitial Biofilm Assay and Biofilm Migration}

A tunnel-based interstitial biofilm assay was developed to mimic microbial biofilm growth on drivelines within a subcutaneous tissue tunnel (Figure 1,C). Agar tunnels were cast in a 6-well microplate by placing a 2.5-cm smooth section of the driveline onto $3 \mathrm{~mL}$ of melted MullerHinton agar or Roswell Park Memorial Institute 1640 agar in a microwell and removing it from the solid agar after 1 hour. The smooth or velour driveline cut-outs with adhered microorganisms, prepared as described in the adherence assay, were placed in the tunnel, with the convex surface touching the base of the tunnel (Figure 1,C). The tunnel was later covered by a lid prepared with $2 \mathrm{~mL}$ of the same agar. Biofilms were allowed to grow for 72 hours at $37^{\circ} \mathrm{C}$ and were assessed using viable counts. Migration of the tunnel-based interstitial biofilms was assessed using a method modified from Gloag and colleagues ${ }^{14}$ and is described in the Appendix E1.

\section{Adhesive Surface Coating}

A silicone (polydimethylsiloxane [PDMS]) (MED-1511, NuSil, Carpinteria, Calif) and a 2-component polyurethane-based adhesive (Loctite M-11FL) were used to prepare glue-coated surfaces. MED-1511 is the adhesive used by HeartWare International Ltd to attach the velour to the smooth tube. Loctite M-11 has been frequently used to bond medical devices and was 


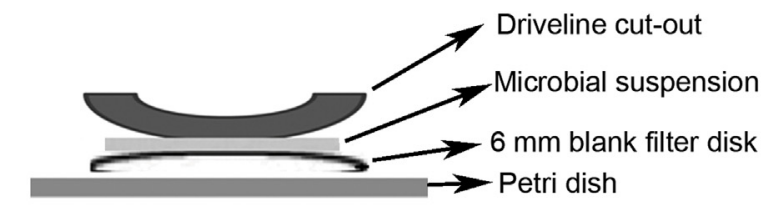

A
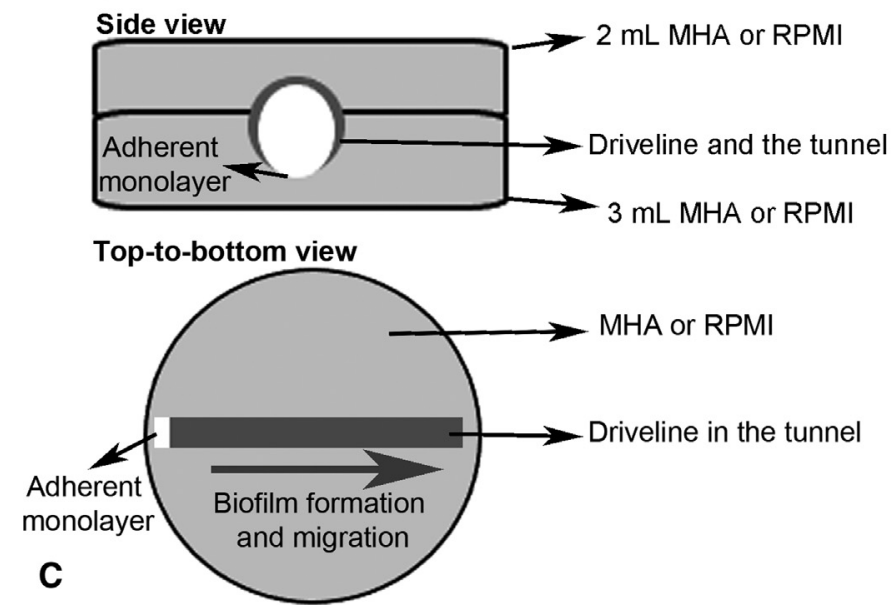

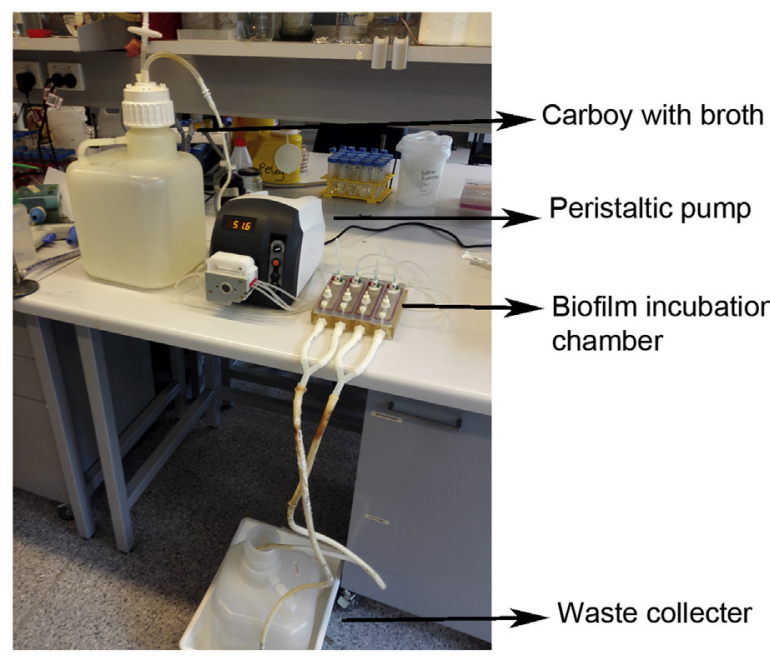

B

FIGURE 1. In vitro biofilm and adherence assays used in this study. A, Schematic description of the early adherence assay. B, The drip-flow biofilm reactor setup. Different components are labeled. C, Schematic description of the tunnel-based interstitial biofilm assay and the biofilm migration assay. For the tunnel-based interstitial biofilm assay, early adherent monolayers were pre-established on driveline cut-outs in a petri dish (A). The driveline cut-out with an adherent monolayer was placed into an enclosed agar tunnel and incubated for 72 hours in a $37^{\circ} \mathrm{C}$ humid chamber. For the biofilm migration assay, early adherent monolayers were grown on 1 end of a $2.5-\mathrm{cm}$ smooth driveline tube. The driveline smooth tube with adherent monolayers was transferred into an enclosed agar tunnel and incubated for 72 hours in a $37^{\circ} \mathrm{C}$ humid chamber. Biofilm growth and migration were monitored using an inverted time-lapse phase contrast microscope. MHA, Muller-Hinton agar; RPMI, Roswell Park Memorial Institute.

included in this study for comparison purposes. Methods for assessing impact of adhesive on biofilm formation are described in the Appendix E1.

\section{Explanted HVAD Drivelines From Patients}

Explanted drivelines from 2 patients undergoing cardiac transplantation were obtained with informed consent. Neither patient had a driveline infection at the time of surgery. The Ethics Review Board of the Alfred Hospital, Melbourne, approved the study (Project 54/16). Explanted drivelines were aseptically sectioned into small pieces that represented the full length of the driveline including the smooth and velour sections. The driveline position of the skin exit site was marked at the time of surgery. All pieces underwent microbiological culture after sonication and SEM. For SEM, driveline pieces were prepared and imaged as previously described, but without dehydration treatment with hexamethyldisilazane. ${ }^{15}$

\section{Statistical Analyses}

For each assay, a minimum of 3 biological replicates were performed for each microorganism. To analyze differences in biofilm formation under each condition, a 1-way analysis of variance or nonparametric method (Mann-Whitney) was performed with Minitab 16 for Windows depending on the data distribution.

\section{RESULTS}

\section{Microbial Adherence and Biofilm Formation on Drivelines}

To assess early adherence and mature biofilm formation of the 4 most common pathogens causing VAD-associated infections on the different components of HVAD drivelines, an in vitro adherence assay was developed and the drip-flow biofilm reactor assay was adopted. The latter was to mimic the air-surface interface of the driveline exit wound site. As shown in Figure 2, $A$, all 4 microorganisms were able to adhere to the smooth and velour sections of the driveline within 2 hours, with all except for $P$ aeruginosa showing greater adherence to the smooth section. This translated into all microorganisms establishing monolayers or multilayer biofilms over 72 hours (Figure 2,B), with SEM images confirming attachment of microorganism clusters (Figure 2, $C$ ). All except $S$ epidermidis showed significantly greater biofilms on the velour compared with the smooth section of the driveline (Figure 2, B). Species-dependent variation in biofilm production was seen, with $P$ aeruginosa and $C$ albicans forming greater biofilms on both smooth and velour driveline sections compared with $S$ aureus and $S$ epidermidis (Figure 2, $B$ and $C$ ).

\section{Driveline Design and Manufacturing Impact on Microbial Biofilm Formation}

To understand further why microorganisms formed greater biofilms on the velour section of HVAD drivelines, the structure of the velour was determined, with the 
Early adherence

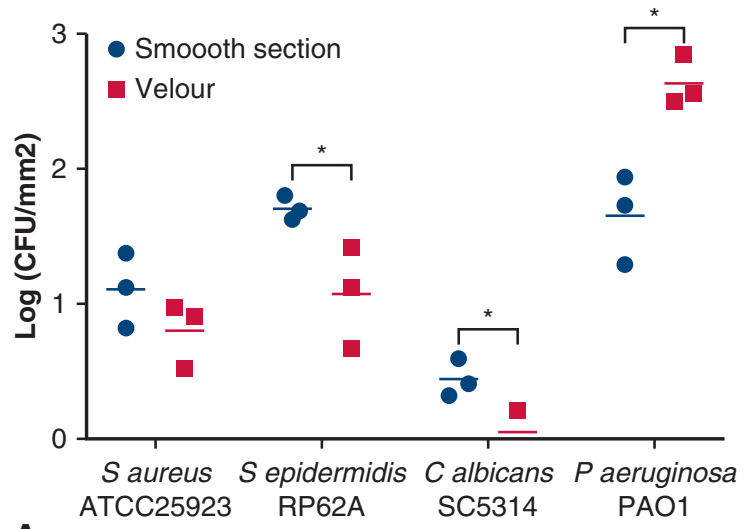

A

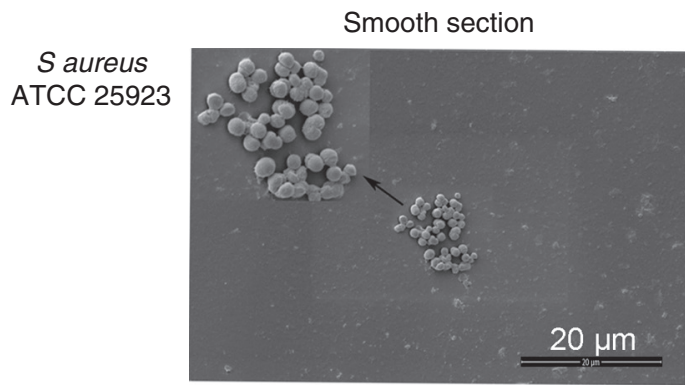

$S$ epidermidis RP62A

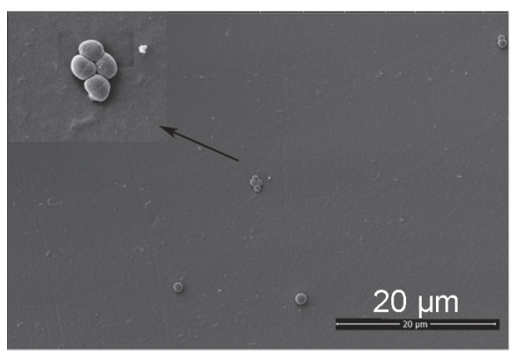

C albicans

SC5314

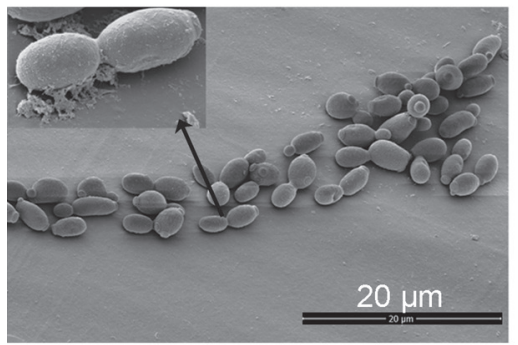

$P$ aeruginosa PAO1

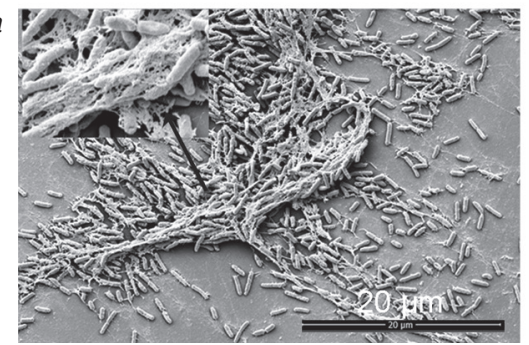

C

FIGURE 2. Early adherence and biofilm formation of microorganisms on HVAD driveline components. A, Early adherence of microorganisms on the driveline smooth and velour sections, as determined by viable counts. All 4 microorganisms were able to adhere to the smooth and velour sections of
Mature biofilm formation
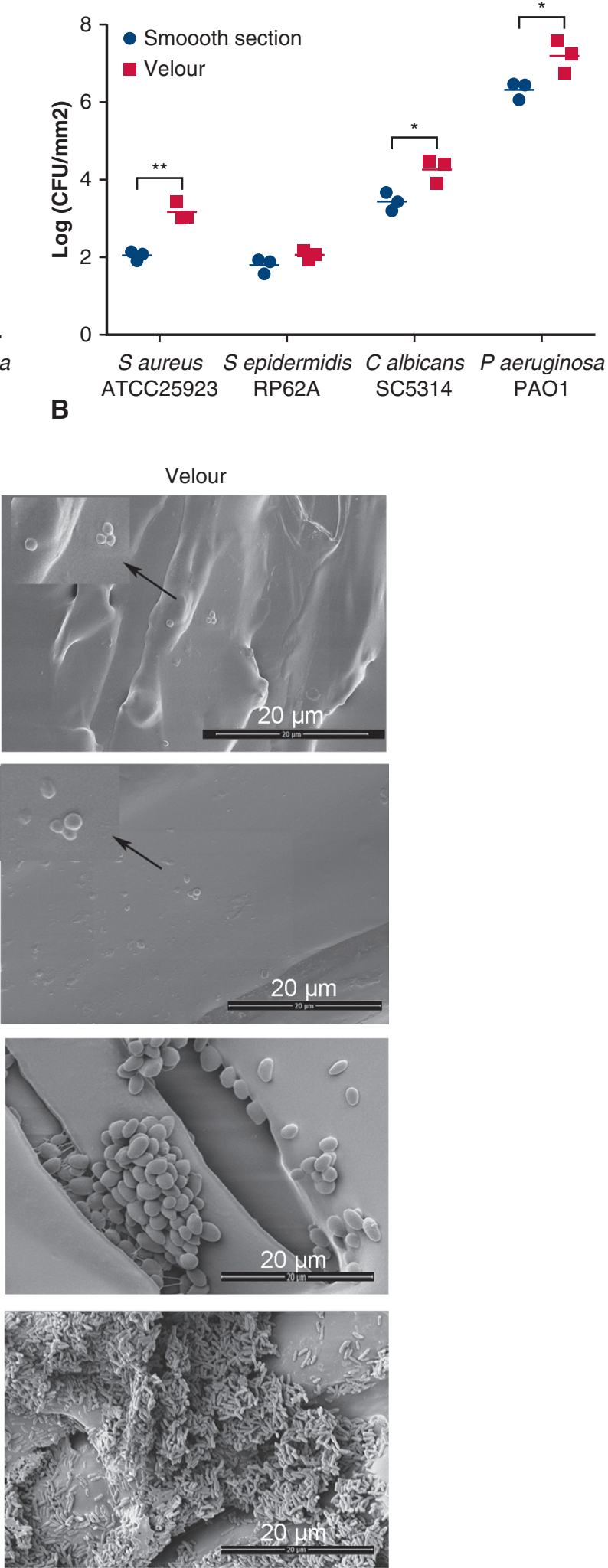
hypothesis that it provided a scaffold that facilitated biofilm formation. SEM imaging of the velour driveline section showed an intricate 3-dimensional structure (Figure 3, A). Notably, the driveline velour, particularly the inter-fiber space, supported biofilm formation of $S$ aureus, C albicans, and $P$ aeruginosa, with microorganisms being seen from the outer surface down to the smooth section-velour interface (Figure $3, C$ ). $P$ aeruginosa and $C$ albicans were able to colonize the entire velour cross-section, with robust biofilms formed on the outer surface and less biofilms or sporadic cell attachment on the inner surface and the supporting tube surface (Figure 3,C). These data showed that the 3-dimensional structure of the HVAD driveline velour may provide a scaffold for microbial biofilm formation.

Recent studies have suggested that microbial attachment and biofilm formation are directly associated with the surface chemistry of biomaterials. ${ }^{16}$ By using XPS analysis, it was determined that the surface of the smooth driveline section resembled polyurethane (Table E1). All components of the smooth section, including oxygen, nitrogen, and carbon, were in agreement with those expected for polyurethane (NIST XPS database, accessed December 12, 2017). Small amounts of PDMS (also known as silicone, $12.9 \%$ ) were also identified using samples from the smooth section. Despite the manufacturer (HeartWare International Inc) confirming that the velour section was made of polystyrene, our analyses showed it was most consistent with PDMS, which is used as an adhesive to adhere the smooth and velour sections together. Notably, it was observed that $P$ aeruginosa had a predilection for attaching and forming biofilms on the adhesive found on the fibers of the velour, with less observed on the clean areas of the fibers (Figure 4, A).

To determine the impact of this PDMS contamination on biofilm formation on HVAD drivelines, we quantitatively and qualitatively assessed biofilm formation of all studied microorganisms on polypropylene surfaces coated with PDMS or polyurethane. For $S$ epidermidis, $P$ aeruginosa, and $C$ albicans, biofilm formation was significantly greater on the PDMS-coated surface compared with the polyurethane-coated surface (Figure 4, B). The polyurethane-coated surfaces showed a striking reduction in biofilm formation by $C$ albicans (Figure $4, B$ and $C$ ).
These data, along with the SEM images (Figure 4, $A$ ), provide experimental evidence that the PDMS glue, which contaminates the HVAD drivelines, can promote biofilm formation.

\section{A Nutrient-Rich Tunnel Supports Robust Biofilms on Drivelines}

Despite staphylococci being major causes of driveline infections, we observed relatively small amounts of biofilms using the drip flow biofilm reactor that mimics the airsurface interface of a chronic wound at the driveline exit site (Figure 2, B). We hypothesized that the subcutaneous tract provides a nutrient-rich tunnel that facilitates a more robust interstitial biofilm compared with that observed using the exit site model. Interstitial biofilms are a relatively new description, often forming in the interstitial space between tissues and a device surface, and are characterized by the formation of networks of interconnected trails and rapid microbial migration along the interface. ${ }^{14} \mathrm{We}$ developed a tunnel-based interstitial biofilm assay to mimic the subcutaneous environment of a driveline tunnel (Figure 1, $C)$ and evaluated biofilm formation. Notably, the biofilms for staphylococci were 500 to 10,000 times higher in the tunnel-based model compared with our exit site model, reaching densities of approximately $5 \times 10^{4}$ to $10^{6} \mathrm{CFU}$ per $\mathrm{mm}^{2}$ (Figure 5). Similar densities were also achieved for Paeruginosa and $C$ albicans (Figure 5). These data support previous literature showing that environmental factors play a strong role in biofilm formation of staphylococci, ${ }^{17}$ and the presence of the driveline within a nutrient-rich tunnel that mimics the subcutaneous tract augments biofilms.

\section{Biofilm Migration Within a Driveline Tunnel}

Our current mechanistic model for invasive driveline infection is that microorganisms adhere to the external smooth section of the driveline, form biofilms, and then migrate into the subcutaneous tract and form robust interstitial biofilms on the intricate network of the velour section within a nutrient-rich environment. We characterized the ability of biofilms to migrate along the driveline using our tunnel-based interstitial biofilm model and time-lapse imaging. The migration distance was measured at 24hour intervals, and we found that $S$ aureus, $C$ albicans,

\footnotetext{
the driveline within 2 hours. B, Biofilm formation on the driveline smooth and velour sections using a drip-flow biofilm reactor, assessed by viable counts. All microorganisms established monolayers or multilayer biofilms over 72 hours. Each dot represents 1 biological repeat, which was the average measurement of 3 technical replicates. All except Staphylococcus epidermidis showed significantly greater biofilms on the velour compared with the smooth section of the driveline (Staphylococcus aureus, $103.17 \pm 0.23 \mathrm{CFU} / \mathrm{mm}^{2}$ versus $102.05 \pm 0.12 \mathrm{CFU} / \mathrm{mm}^{2}, P<.01$; Candida albicans, $104.27 \pm 0.32 \mathrm{CFU} / \mathrm{mm}{ }^{2}$ versus $103.44 \pm 0.24 \mathrm{CFU} / \mathrm{mm}^{2}, P<.05 ;$ Pseudomonas aeruginosa $107.20 \pm 0.41 \mathrm{CFU} / \mathrm{mm}^{2}$ versus $\left.106.33 \pm 0.23 \mathrm{CFU} / \mathrm{mm}^{2}, P<.05\right) . P$ aeruginosa and $C$ albicans formed greater biofilms on both smooth and velour driveline sections compared with $S$ aureus and $S$ epidermidis. C, SEM of microbial biofilm formation on the driveline smooth and velour sections using a drip-flow biofilm reactor. Dehydrated extracellular polymeric substance (biofilm matrix) was observed in biofilms formed by $P$ aeruginosa and $C$ albicans on both smooth (inserts) and velour sections. $* P<.05, * * P<.01, * * * P<.001$ refer to methods for statistical analysis. Scale bar $=20 \mu \mathrm{m}$.
} 

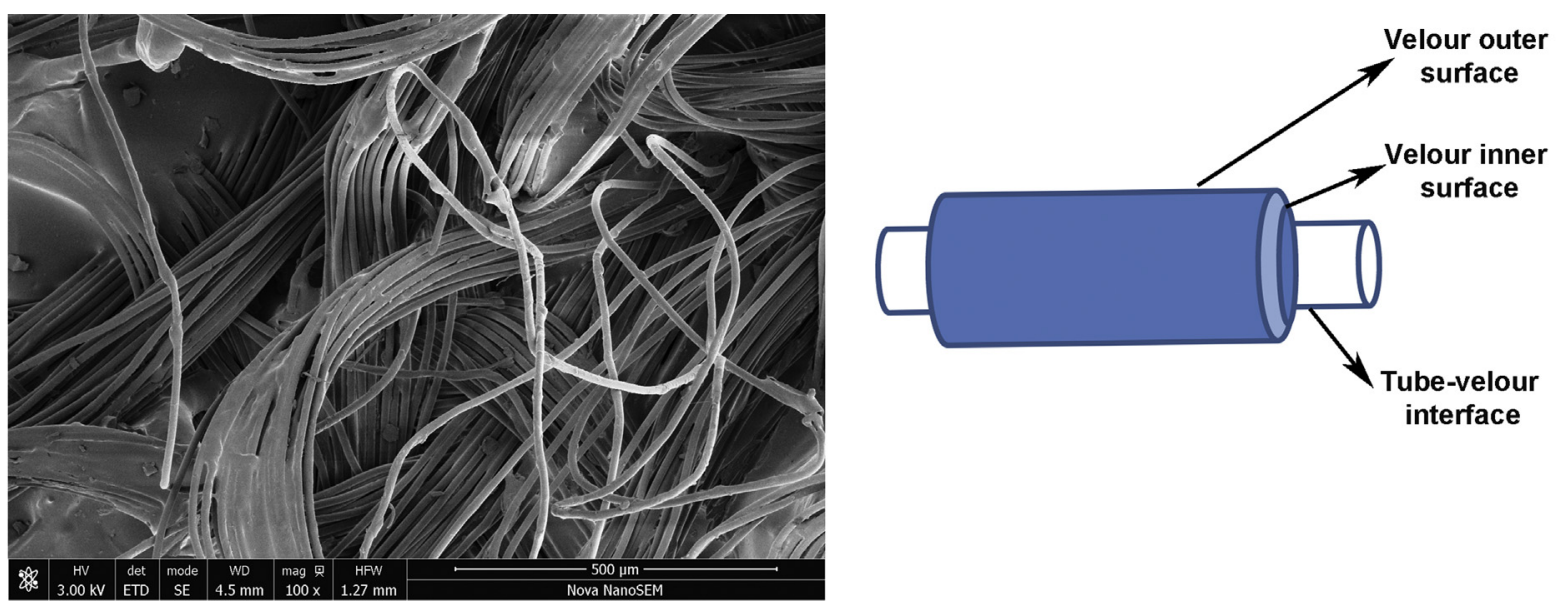

A

B

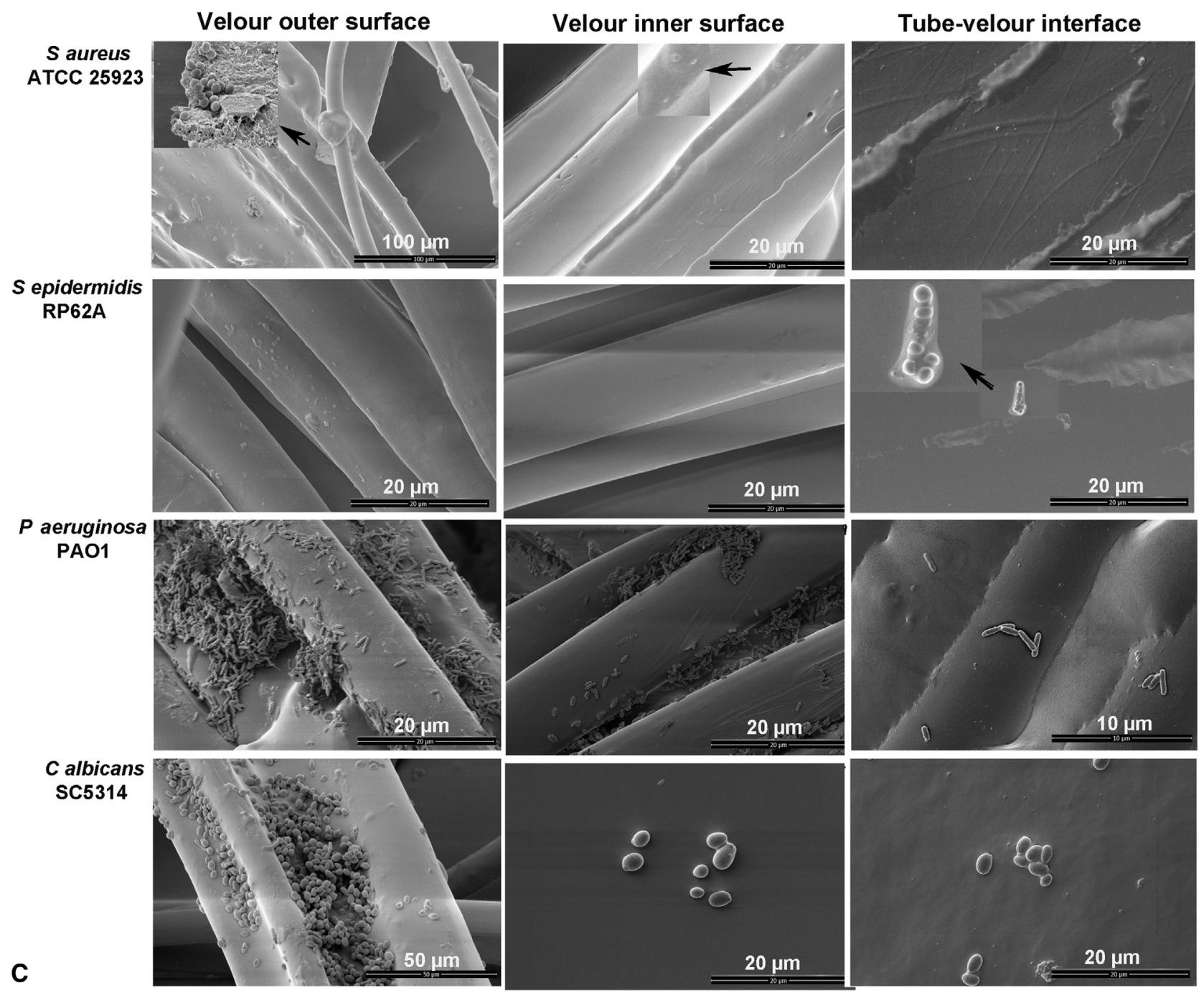

FIGURE 3. SEM of microbial biofilms formed on the HVAD driveline. A, An intricate 3-dimensional structure of the velour section of the HVAD driveline. B, Schematic description of an HVAD driveline and the surfaces that were imaged. C, SEM of microbial biofilms formed on the different surfaces of the velour section of the HVAD driveline using the drip-flow biofilm reactor. 

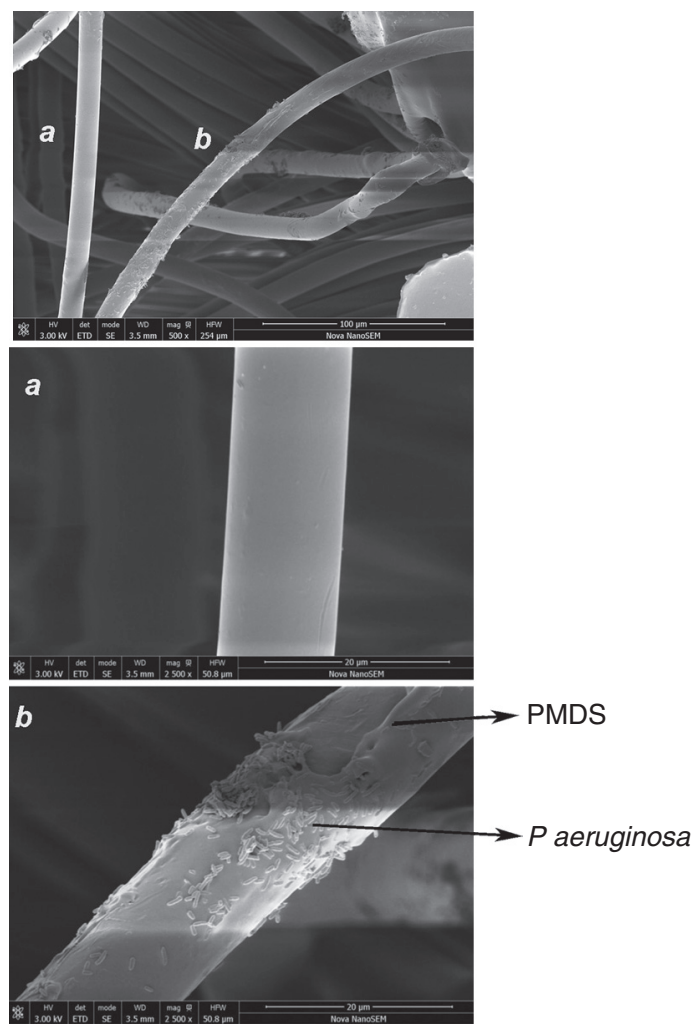

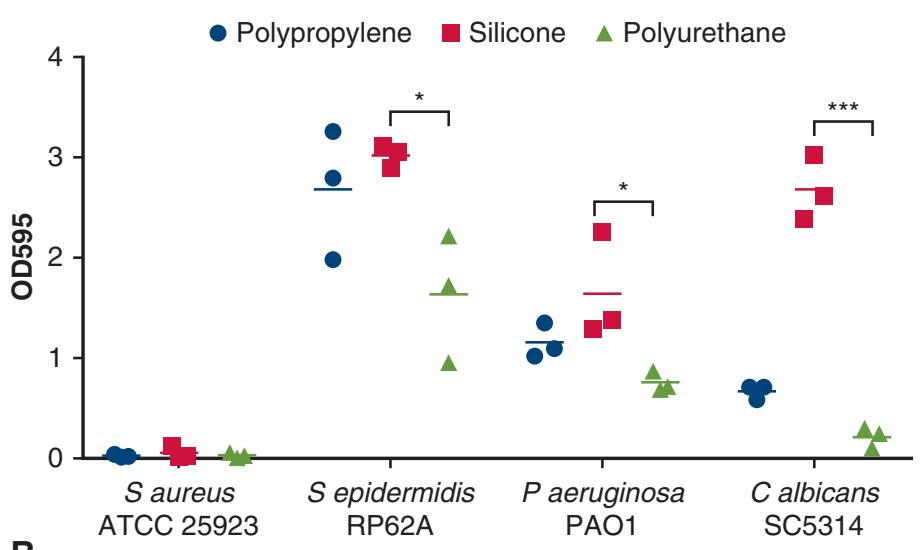

B

A

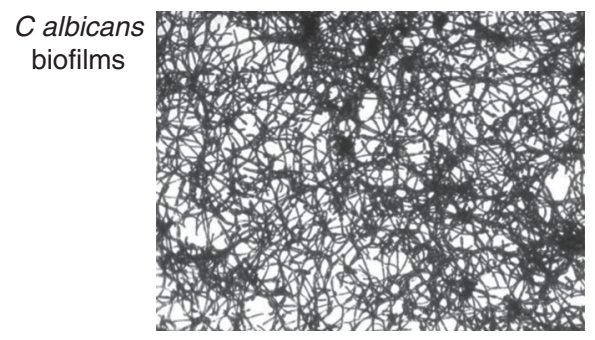

Polypropylene control

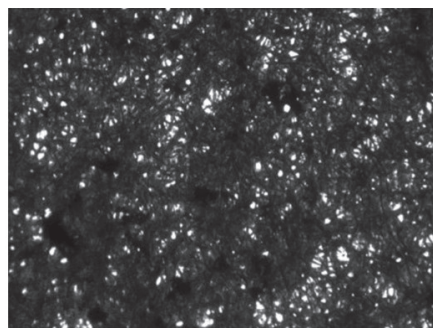

Silicone adhesive

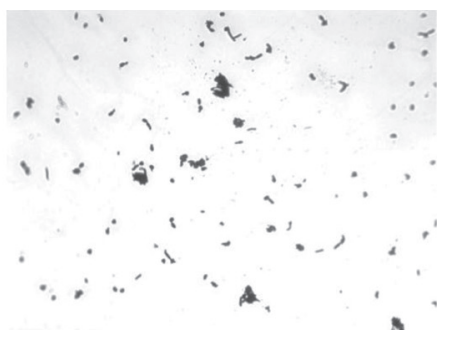

Polyurethane adhesive

C

FIGURE 4. PDMS adhesive enhances biofilm formation on HVAD drivelines. A, SEM of biofilm formation by P aeruginosa on the clean velour fiber (a) and a fiber covered with PDMS (b). No biofilm was observed on the clean velour fiber without PDMS attached. B, Biofilm formation on biomaterial surface coated with PMDS and polyurethane adhesives (for comparison purpose). Biofilms were cultured in 96-well microplates precoated with different adhesives, stained with crystal-violet, and examined by reading their optical density at $600 \mathrm{~nm}$, as described before. ${ }^{7}$ Each $d o t$ represents 1 biological repeat, which was the average measurement of 3 technical replicates. Statistical analysis was performed between the readings of polyurethane-coated surfaces and PDMScoated surfaces. $* P<.05,{ }^{*} P<.01,{ }^{* * *} P<.001$ refer to methods for statistical analysis. C, Biofilms formed by $C$ albicans SC5314 on polypropylene surfaces coated with silicone adhesive or polyurethane adhesive were qualitatively assessed with light microscopy. PDMS, Polydimethylsiloxane.

and $S$ epidermidis readily migrated along the driveline surface and reached almost the full length within 72 hours (Figure 6, A). In contrast, $P$ aeruginosa exhibited no added migration beyond the tunnel only control (Figure 6, A). Phase-contrast images supported these quantitative data (Figure 6, B). These data demonstrate that microbial biofilms can migrate along HVAD driveline surfaces, supporting our mechanistic model of invasive driveline infections.

\section{Explanted Patient HVAD Drivelines Show Micro- Gaps Between Velour Fibers}

To evaluate the in vivo morphology of drivelines, 2 explanted drivelines from separate patients were examined (Figure 7, A). Apart from a very low density (3-4 CFU/ $\mathrm{mm}^{2}$ ) of $S$ epidermidis from the skin exit site section of 1 driveline, no other microorganisms were cultured. Notably, high-resolution SEM showed inadequate tissue in-growth of the entire length of the velour section of the driveline, 


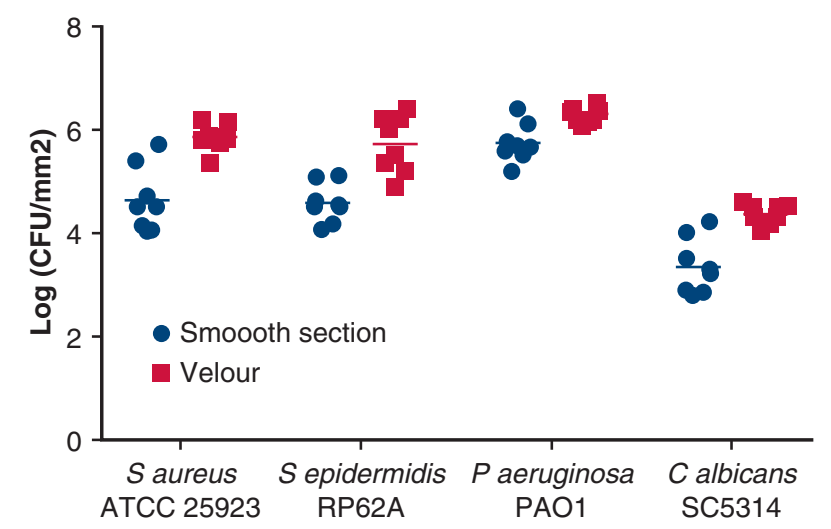

FIGURE 5. Formation of interstitial biofilms in a driveline tunnel for the velour and smooth sections of the HVAD driveline using a tunnel-based biofilm assay, assessed by viable counts. Six biological repeats were carried out. Biofilm formation of staphylococci were 500 to 10,000 times greater in the tunnel-based biofilm model compared with our exit site model using the drip-flow biofilm reactor.

which appeared to be more pronounced for sections closer to the skin exit site (Figure 7, B, velour section V2). Micro-gaps between the velour fibers or between the velour and its underlying smooth tube were constantly seen (Figure 7, B).

\section{DISCUSSION}

Biofilm formation on VAD drivelines is one of the most important factors contributing to the establishment of VAD-associated infections. ${ }^{7}$ The specific growth mode of biofilms renders infection less responsive to conventional antimicrobial treatments and surgical interventions and is believed to be the cause of the persistence of VADassociated infections. More experimental data on the formation, regulation, and migration of microbial biofilms on VAD drivelines is required to develop more effective infection mitigation strategies. In this study, we used in vitro assays to examine the interactions among 3 major factors involved in the pathogenesis of driveline infections, including the pathogens, the driveline components, and the environments that represent the host context, and further assessed the clinical significance of our findings by studying explanted drivelines from patients with VADs. We have shown that (1) clinically relevant microorganisms formed biofilms on both the smooth and velour sections of HVAD drivelines; (2) factors related to driveline design and manufacturing, such as the intricate velour network and the PDMS adhesive promote biofilm formation; (3) the presence of the driveline tunnel may be a contributing factor to biofilm formation; (4) biofilm migration in a speciesdependent manner occurs along drivelines within a tunnel environment; and (5) micro-gaps were found around the velour of explanted patient drivelines and may play a significant role in invasive VAD infections.
To best study the impact of anatomy and driveline positioning in patients on driveline infections, we adopted or developed various assays to best mimic these environments. Using an early adherent assay and drip-flow biofilm reactor to mimic the driveline exit site, we found all studied microorganisms were able to attach and form biofilms on the smooth and velour sections of HVAD drivelines, with $S$ aureus and $S$ epidermidis forming lower biomass. However, once the subcutaneous driveline tract was modeled, robust staphylococcal biofilms were observed. The drip-flow biofilm reactor assay and the tunnel-based interstitial biofilm assay differ in nutrient and oxygen supply, as well as spatial containment, with our results showing the significance of a nutrient-rich tunnel in the pathogenesis of driveline infections.

The most common microorganisms causing VADassociated infections differ slightly depending on the site of infection., ${ }^{9-22}$ For example, $P$ aeruginosa was the second most common microorganism involved in driveline exit site infections, but was less frequently isolated from deeper tissue infections. ${ }^{9,18-22}$ Features of microbial biofilms that determine their migration along the driveline-tunnel interface might explain the change in causative pathogens of the different infection sites of VADs/drivelines. In keeping with this hypothesis, we showed that $P$ aeruginosa biofilms demonstrated almost no migration along the HVAD driveline within a nutrientrich tunnel. The poor migration of $P$ aeruginosa might be related to the restricted access to oxygen at the drivelinetunnel interface, because expansion of Paeruginosa biofilm has been found to be mediated by its twitching motility that switches to low speed under oxygen depletion. ${ }^{14,23}$ The slower biofilm migration of $S$ epidermidis relative to that of $S$ aureus found in our in vitro study is also in agreement with that reported in an in vivo study by Toba and colleagues. ${ }^{7}$ Together, these data suggest that speciesdependent pathogenic mechanisms of biofilm formation under various environmental conditions likely explain the causative microorganisms of the different VAD-associated infection types.

The impact of driveline materials and surface chemistry on driveline infections remains poorly understood. Surface chemistry of biomaterials is one of the key determinants of device-related microbial infections. ${ }^{24,25}$ In general, microorganisms have a preference to adhere to siliconebased biomaterials compared with Teflon or polyurethane. ${ }^{24,25}$ This is supported by recent studies that found the polyurethane HVAD driveline had a lower incidence of infection compared with silicone-based drivelines used by other VAD systems, such as the HeartMate II (St Jude, Pleasanton, Calif), although other factors such as the size of drivelines might have played a role. ${ }^{26,27}$ However, despite the HeartWare's anti-infective effort by using polyurethane and polystyrene for driveline manufacturing, our 

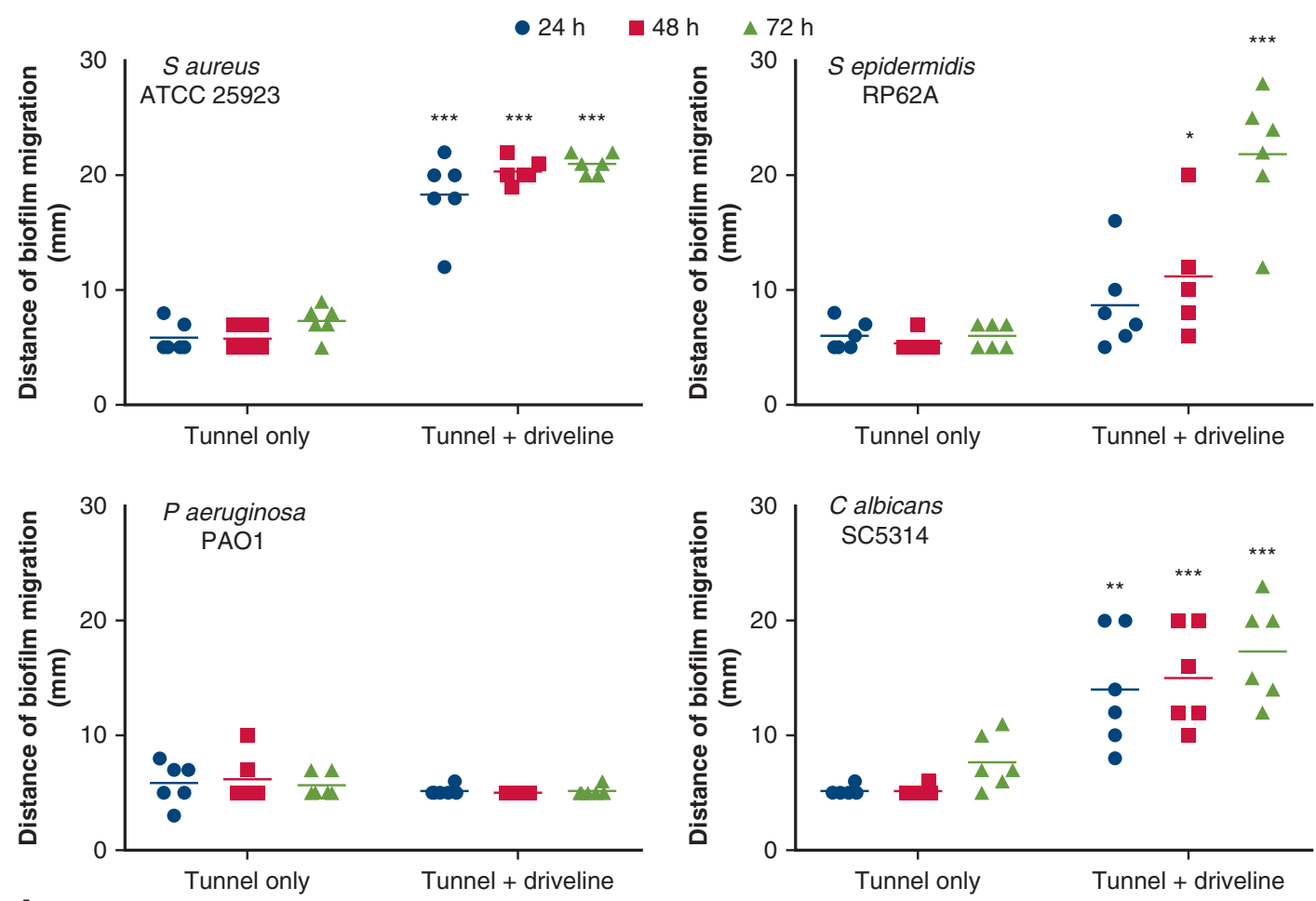

A

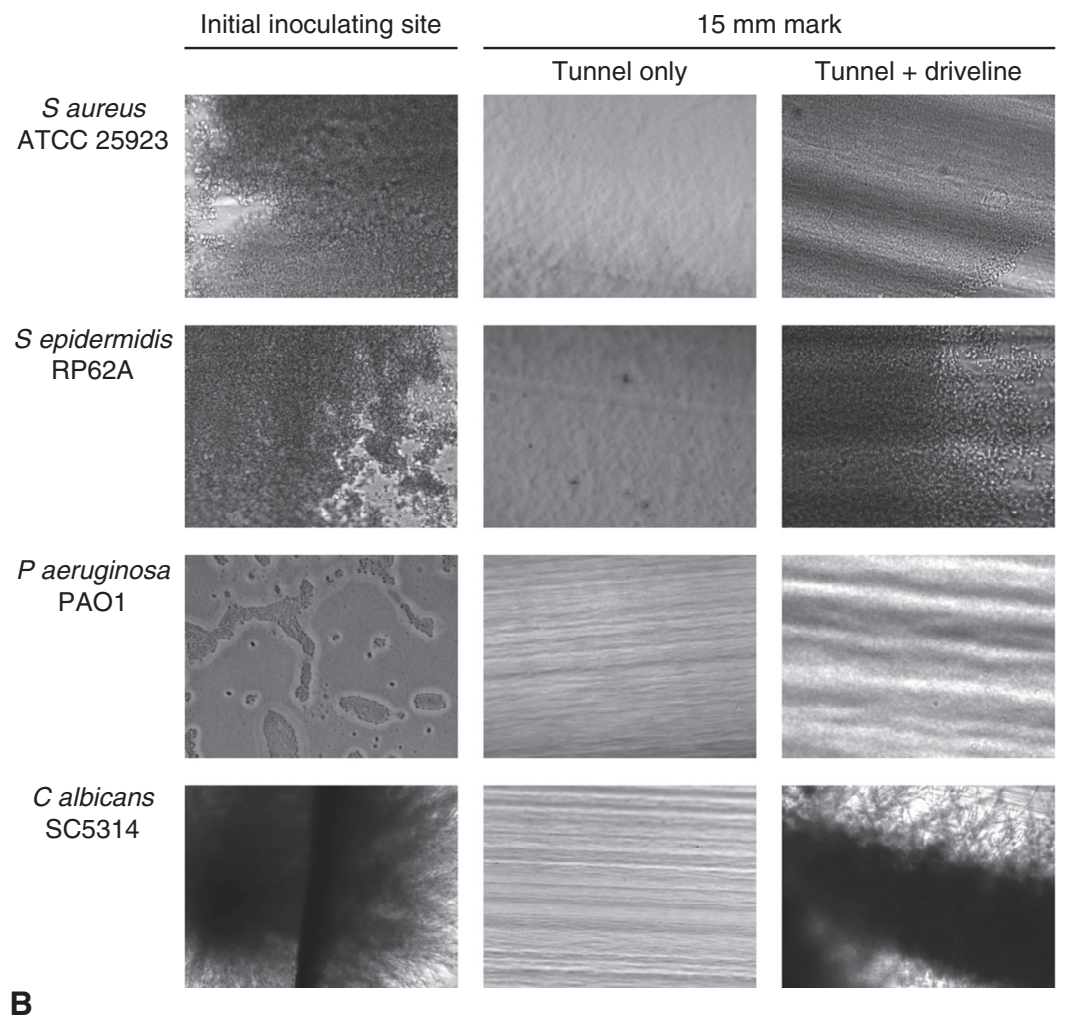

FIGURE 6. Biofilm migration at the driveline-tunnel interface. A, Biofilm migration was quantitatively assessed by measuring migration distances at 24-hour intervals for the 4 pathogens. S aureus, Calbicans, and S epidermidis, but not $P$ aeruginosa readily migrated along the driveline surface and reached almost the full length within 72 hours. Six biological repeats were carried out. $* P<.05$, $* * P<.01, * * * P<.001$ refer to methods for statistical analysis. B, Biofilm migration was qualitatively examined at the $15-\mathrm{mm}$ mark of the driveline (away from the initial inoculating site) by using a phase-contrast microscope after 48-hour incubation. 

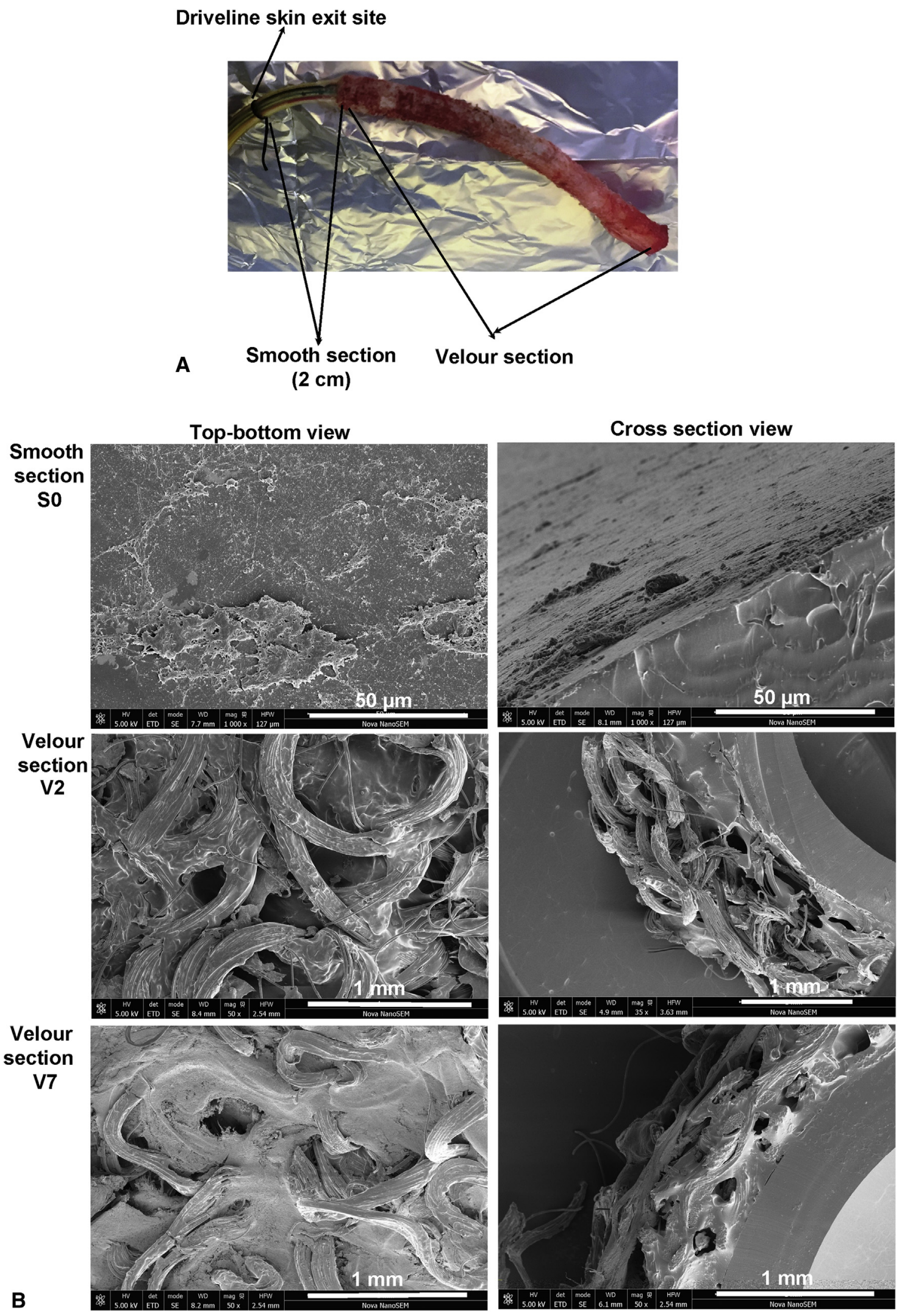

FIGURE 7. SEM of a HVAD driveline explanted from a patients with a VAD. A, HVAD driveline explanted from a patients with VAD undergoing cardiac transplantation. B, SEM of velour sections of the explanted HVAD driveline. The velour of the explanted driveline was aseptically sectioned into 8 pieces 


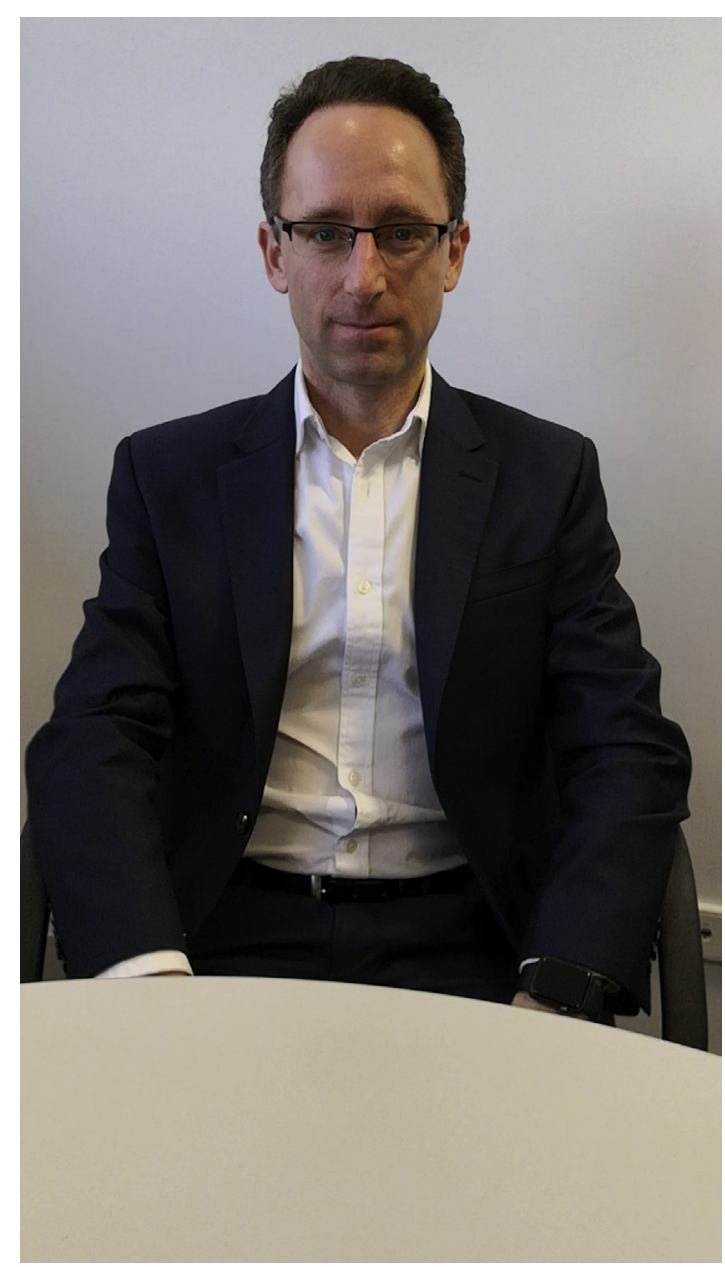

VIDEO 1. This study identified key factors involved in biofilm formation and migration on VAD drivelines, including surface chemistry and design of the driveline, and the presence of a nutrient-rich subcutaneous tunnel. These findings lay the foundation for preventative strategies including improved tissue integration into the velour, altered driveline manufacturing, and surface-attached antimicrobials. Video available at: https://www.jtcvs.org/article/S0022-5223(19)30556-2/fulltext.

study found contamination of the HVAD drivelines (smooth and velour sections) by PDMS that originated from the silicone adhesive used in the manufacturing process. It is well established that lower molecular PDMS components can migrate along material surfaces in hydrophobic environments (eg, during storage in air), which might have led to PDMS contamination on all surfaces of the HVAD driveline. These data highlight the relevance of driveline surface characteristics to the risks of microbial biofilm formation and subsequent infection, and indicate a potential manufacturing intervention that could reduce VADassociated infections.

Adding velour to the driveline to enhance its tissue interaction and stability has been widely adopted by VAD manufacturers. We dissected the driveline velour after microorganism inoculation and found that microorganisms colonized the entire 3-dimensional network structure. Gaps were observed between the velour fibers, with microorganisms observed within the gaps attached to the complex network. To support these findings, high-resolution SEM of HVAD drivelines explanted from patients with VADs was performed and found numerous micro-gaps throughout the length of the velour, supporting the hypothesis that micro-gaps within the velour network may facilitate pathways for biofilm migration and invasive infection. These data also suggest that augmented tissue integration into the complex velour network may be a strategy to hinder biofilm migration along drivelines.

\section{Study Limitations}

One obvious limitation of this study is that our in vitro assays do not comprehensively reflect the complexity of in vivo conditions in the human, such as the presence of local inflammatory responses at the skin exit site and micro-trauma caused by mechanical instability of the implanted drivelines; however, through use of specialized biofilm assays, we provide important initial insights into pathogenic mechanisms of driveline infections. Validation of our findings in an animal model of percutaneous driveline infection would be useful. ${ }^{28}$ Our use of explanted patient drivelines also supports the assessment of the human tissue-driveline interface, and further work will follow on examining infected patient drivelines.

\section{CONCLUSIONS}

The current study identified several key factors involved in biofilm formation on VAD drivelines and laid a foundation for the development of discrete prevention strategies. Apart from established targets for preventing driveline infections such as exit site wound care, our work supports interventions that target the surface chemistry and materials of the driveline, and interventions that would prevent biofilm migration within the nutrient-rich subcutaneous tunnel, which may include improved tissue integration into the velour, altered velour surface architecture, and surfaceattached antimicrobials (Video 1).

and designated as V1 to V8. Section V1 adjoined the smooth tube section at exit site, and section V8 was adjacent to the VAD pump. Tissue in-growth was seen for the entire driveline velour studied, with velour sections close to the VAD (section V8 as a representative) showing a better tissue integration than that close to the skin exit site (section V2 as the representative). Micro-gaps were constantly found at the driveline-tissue tract interface (velour external surface), within the velour, and at the velour-smooth tube interface. Only analytic results of the driveline from 1 patient are shown, because they are representative of both explanted drivelines. 


\section{Conflict of Interest Statement}

D.M. is a proctor for Abbott implantation of the HeartMate III VAD. All other authors have nothing to disclose with regard to commercial support.

The authors acknowledge financial assistance provided by the Alfred Hospital Foundation, Monash Institute of Medical Engineering, the Department of Infectious Diseases, and Department of Microbiology, Monash University, Australia. Publication of this article was also supported by the National Health and Medical Research Council (1079421) CRE ACTIONS. The contents of the published material are solely the responsibility of the Alfred Hospital and Monash University or individual authors and do not reflect the views of National Health and Medical Research Council. A.Y.P also acknowledges an Australian National Health and Medical Research Council Practitioner Fellowship (APP1117940).

\section{References}

1. Oz MC, Gelijns AC, Miller L, Wang C, Nickens P, Arons R, et al. Left ventricular assist devices as permanent heart failure therapy: the price of progress. Ann Surg. 2003;238:577-83.

2. Stulak JM, Davis ME, Haglund N, Dunlay S, Cowger J, Shah P, et al. Adverse events in contemporary continuous-flow left ventricular assist devices: a multiinstitutional comparison shows significant differences. J Thorac Cardiovasc Surg. 2016;151:177-89.

3. Tsiouris A, Paone G, Nemeh HW, Borgi J, Williams CT, Lanfear DE, et al. Short and long term outcomes of 200 patients supported by continuous-flow left ventricular assist devices. World J Cardiol. 2015;7:792-800.

4. Cagliostro B, Levin AP, Fried J, Stewart S, Parkis G, Mody KP, et al. Continuous-flow left ventricular assist devices and usefulness of a standardized strategy to reduce drive-line infections. J Heart Lung Transplant. 2016;35: 108-14.

5. Qu Y, Daley AJ, Istivan TS, Garland SM, Deighton MA. Antibiotic susceptibility of coagulase-negative staphylococci isolated from very low birth weight babies: comprehensive comparisons of bacteria at different stages of biofilm formation. Ann Clin Microbiol Antimicrob. 2010;9:16.

6. Nienaber JJ, Kusne S, Riaz T, Walker RC, Baddour LM, Wright AJ, et al. Clinical manifestations and management of left ventricular assist device-associated infections. Clin Infect Dis. 2013;57:1438-48.

7. Toba FA, Akashi H, Arrecubieta C, Lowy FD. Role of biofilm in Staphylococcus aureus and Staphylococcus epidermidis ventricular assist device driveline infections. J Thorac Cardiovasc Surg. 2011;141:1259-64.

8. Herrmann M, Weyand M, Greshake B, von Eiff C, Proctor RA, Scheld HH, et al. Left ventricular assist device infection is associated with increased mortality but is not a contraindication to transplantation. Circulation. 1997;95:814-7.

9. Nienaber J, Wilhelm MP, Sohail MR. Current concepts in the diagnosis and management of left ventricular assist device infections. Expert Rev Anti Infect Ther. 2013;11:201-10.

10. Qu Y, Locock K, Verma-Gaur J, Hay ID, Meagher L, Traven A. Searching for new strategies against polymicrobial biofilm infections: guanylated polymethacrylates kill mixed fungal/bacterial biofilms. J Antimicrob Chemother 2016;71:413-21.

11. Lipp C, Kirker K, Agostinho A, James G, Stewart P. Testing wound dressings using an in vitro wound model. $J$ Wound Care. 2010;19:220-6.

12. Woods J, Boegli L, Kirker KR, Agostinho AM, Durch AM, Delancey Pulcini E, et al. Development and application of a polymicrobial, in vitro, wound biofilm model. J Appl Microbiol. 2012;112:998-1006.

13. Goeres DM, Hamilton MA, Beck NA, Buckingham-Meyer K, Hilyard JD, Loetterle LR, et al. A method for growing a biofilm under low shear at the air-liquid interface using the drip flow biofilm reactor. Nat Protoc. 2009;4:783-8.

14. Gloag ES, Turnbull L, Huang A, Vallotton P, Wang H, Nolan LM, et al. Self-organization of bacterial biofilms is facilitated by extracellular DNA. Proc Natl Acad Sci U S A. 2013;110:11541-6.

15. Uwamahoro N, Qu Y, Jelicic B, Lo TL, Beaurepaire C, Bantun F, et al. The func tions of Mediator in Candida albicans support a role in shaping species-specific gene expression. PLoS Genet. 2012;8:e1002613.

16. Hook AL, Chang C-Y, Yang J, Luckett J, Cockayne A, Atkinson S, et al. Combinatorial discovery of polymers resistant to bacterial attachment. Nat Biotech. 2012;30:868-75.

17. Kavanaugh JS, Horswill AR. Impact of environmental cues on staphylococcal quorum sensing and biofilm development. J Biol Chem. 2016;291: 12556-64.

18. Rubinfeld G, Levine JP, Reyentovich A, DeAnda A, Balsam LB. Management of rapidly ascending driveline tunnel infection. J Card Surg. 2015;30:853-5.

19. Schaffer JM, Allen JG, Weiss ES, Arnaoutakis GJ, Patel ND, Russell SD, et al. Infectious complications after pulsatile-flow and continuous-flow left ventricular assist device implantation. J Heart Lung Transplant. 2011;30:164-74.

20. Gordon RJ, Weinberg AD, Pagani FD, Slaughter MS, Pappas PS, Naka Y, et al. Prospective, multicenter study of ventricular assist device infections. Circulation. 2013;127:691-702.

21. Monkowski DH, Axelrod P, Fekete T, Hollander T, Furukawa S, Samuel R. Infections associated with ventricular assist devices: epidemiology and effect on prognosis after transplantation. Transpl Infect Dis. 2007;9:114-20.

22. Gordon RJ, Quagliarello B, Lowy FD. Ventricular assist device-related infections. Lancet. 2006;6:426-37.

23. Kurre R, Maier B. Oxygen depletion triggers switching between discrete speed modes of gonococcal type IV pili. Biophys J. 2012;102:2556-63.

24. Pascual A. Pathogenesis of catheter-related infections: lessons for new designs. Clin Microbiol Infect. 2002;8:256-64.

25. Lopez-Lopez G, Pascual A, Perea EJ. Effect of plastic catheter material on bacterial adherence and viability. J Med Microbiol. 1991;34:349-53.

26. Tuncer ON, Kemaloglu C, Erbasan O, Golbasi I, Turkay C, Bayezid O. Outcomes and readmissions after continuous flow left ventricular assist device: Heartmate II versus Heartware ventricular assist device. Transplant Proc. 2016;48:2157-61.

27. Haglund NA, Davis ME, Tricarico NM, Keebler ME, Maltais S. Readmissions after continuous flow left ventricular assist device implantation: differences observed between two contemporary device types. ASAIO J. 2015;61:410-6.

28. Arrecubieta C, Toba FA, von Bayern M, Akashi H, Deng MC, Naka Y, et al. SdrF, a Staphylococcus epidermidis surface protein, contributes to the initiation of ventricular assist device driveline-related infections. PLoS Pathog. 2009;5: e1000411.

Key Words: VAD, driveline, biofilms, interstitial biofilm, Staphylococcus aureus, Staphylococcus epidermidis, Pseudomonas aeruginosa, Candida albicans 


\section{APPENDIX E1. MATERIALS AND METHODS Driveline Materials}

To study their surface chemistry and interaction with microorganisms, both the smooth tube and the velour of drivelines were cut into sections of $0.5 \mathrm{~cm}$ in length. Each section was further cut longitudinally into 4 pieces, with each piece of cut-out having a size of approximately $3 \times 5 \mathrm{~mm}^{2}$. The cut-outs were then sterilized with ethylene oxide (Steritech, Victoria, Australia) and used for experiments.

\section{Drip-Flow Biofilm Reactor}

Driveline cut-outs with attached microorganisms, prepared as per the microbial adherence assay, were placed on a filter membrane in the biofilm incubation chamber (Figure 1, B). 10\% Tryptone Soy Broth (TSB, Oxoid Ltd, Basingstoke, UK) as growth media was pumped through the system at $5 \mathrm{~mL} / \mathrm{h} / \mathrm{channel}$. Biofilms were allowed to grow for 72 hours at room temperature. The samples were removed from the chamber and washed 3 times with PBS, followed by viable count assessment as described in the adherence assay. Qualitative imaging was also performed using SEM as previously described. ${ }^{28}$

\section{Biofilm Migration Assay}

Migration of the tunnel based interstitial biofilms was assessed using a method modified from Gloag and colleagues. ${ }^{14}$ Smooth driveline tubes of $2.5 \mathrm{~cm}$ in length were used. After microorganism adherence to 1 end of the driveline using the method described, the driveline tubes were placed into a precasted agar tunnel (Figure 1,C). Biofilm migration was monitored using an inverted time-lapse phase contrast microscope (Leica LX widefield; Wetzlar, Germany) for 72 hours.

\section{X-Ray Photoelectron Spectroscopy Analysis}

XPS analysis was used to characterize the surface chemistry of the driveline material and was performed using an AXIS HSi spectrometer (Kratos Analytical Ltd, Manchester, UK) equipped with a monochromated $\mathrm{Al}-\mathrm{K} \alpha \mathrm{X}$-ray source at a power of $144 \mathrm{~W}(12 \mathrm{~mA}, 12 \mathrm{kV})$. All elements presented were identified from low-resolution survey spectra (acquired at a pass energy of $160 \mathrm{eV}$ ). The atomic concentrations of the detected elements were calculated using integral peak intensities and the sensitivity factors supplied by the manufacturer.

\section{Adhesive Surface Coating and Microplate Biofilm Assay}

A silicone (PDMS)-based adhesive (MED-1511, NuSil, Carpinteria, Calif) and a 2-component polyurethane based adhesive (Loctite M-11FL) were used to prepare glue-coated surfaces. MED-1511 is the adhesive used by HeartWare International Ltd to attach the velour to the smooth tube. Loctite M-11 has been frequently used to bond medical devices and was included in this study for comparison purposes. The adhesives were extracted from the tube and dissolved in tetrahydrofuran ( $\geq 99.9 \%$, Chem-Supply, Gillman, South Australia) to afford a $20 \mathrm{wt} \%$ solution. Two-hundred microliters of each adhesive solution was then pipetted into individual wells of a 96-well polypropylene flat bottom microplate (Greiner, Heidelberg West, Australia) at a minimum of 4 repeats. The plate was then covered and placed in an oven at $40^{\circ} \mathrm{C}$ for 18 hours and then maintained in vacuo for 5 hours $(0.2 \mathrm{mbar}$ $40^{\circ} \mathrm{C}$ ). A thick adhesive coating could be observed both at the bottom and the walls of the microwells. The plates were sterilized by ethylene oxide (Steritech, Victoria, Australia) and used for biofilm quantitative and qualitative assessment, following the method described previously. ${ }^{6}$ 
TABLE E1. Elemental surface concentration and ratios of different HVAD driveline components as determined by X-ray photoelectron spectroscopy*

\begin{tabular}{lcc}
\hline & $\begin{array}{c}\text { Smooth tube } \\
(\text { mean } \pm \text { SD) }\end{array}$ & $\begin{array}{c}\text { Velour section } \\
(\text { mean } \pm \text { SD) }\end{array}$ \\
\hline Atomic\% & & \\
Carbon & $66.3 \pm 2.2$ & $52.9 \pm 1.0$ \\
Oxygen & $19.2 \pm 1.2$ & $25.5 \pm 0.3$ \\
Nitrogen & $1.6 \pm 0.2$ & $0 \pm 0$ \\
Silicone & $12.9 \pm 1.2$ & $21.7 \pm 1.4$ \\
Atomic ratio (X/C) & & \\
O & $0.290 \pm 0.028$ & $0.482 \pm 0.003$ \\
N & $0.025 \pm 0.002$ & $0.000 \pm 0.000$ \\
Si & $0.195 \pm 0.024$ & $0.410 \pm 0.034$ \\
\hline
\end{tabular}

$S D$, Standard deviation. *Detailed methods refer to "Materials and Methods" in Appendix E1. 